\title{
A randomised controlled study of the effect of long chain polyunsaturated fatty acid supplementation on stool hardness during formula feeding
}

\author{
J S Forsyth, S Varma, M Colvin
}

\begin{abstract}
Background-The passage of hard stools is significantly more common in formula fed infants than breast fed infants and this might be the result of differences in fat absorption between breast and formula fed infants. Experimental studies indicate that long chain polyunsaturated fatty acids (LCPUFAs) might influence fat hydrolysis and absorption.

Aim-To investigate the relation of LCPUFA supplementation to stool frequency and consistency during the first 4 months of life.

Design-Double blind, randomised, controlled study of 88 healthy infants.

Results-1905 stools (858 from LCPUFA supplemented infants, 1047 nonsupplemented infants) were examined. The mean (SEM) number of stools passed for each three day study period was significantly less in the LCPUFA group (5.5 (0.3) $v 6.2(0.2) ; p<0.05)$. In both groups, there was a significant reduction in the number of stools passed with age ( $p<0.001)$. During the first 3 months, the mean (SEM) percentage of hard stools passed by infants in the LCPUFA supplemented group was 7.7 (2.1) compared with 19.2 (2.8) for unsupplemented infants $(\mathrm{p}=0.001)$.
\end{abstract}

Conclusion-The prevalence of hard stools is significantly reduced in infants fed a formula that is supplemented with LCPUFAs.

(Arch Dis Child 1999;81:253-256)

Keywords: long chain polyunsaturated fatty acids; formula feeding; hard stools; calcium soaps; randomised controlled trial

During infancy the passage of hard stools can cause considerable distress to both infants and parents. ${ }^{12}$ Stool hardness in infancy is related to the calcium soap content of the stool, ${ }^{3}$ and soaps are formed when residual free fatty acids, especially saturated fats, precipitate with calcium in the intestinal lumen. ${ }^{4}$ Hard stools are more prevalent in formula fed infants, ${ }^{67}$ and it has been suggested that this is because of differences in fat digestion and absorption between breast and formula fed infants. ${ }^{3}$

The more efficient digestion of fat by breast fed infants is thought to be the result of the presence of bile salt stimulated lipase in breast milk, ${ }^{8}$ and also the fact that the chemical structure of breast milk triacylglycerol is more favourable to fat hydrolysis. ${ }^{9-11}$ However, recent experimental studies have indicated that long chain fatty acids may play an important role in fat hydrolysis and absorption. ${ }^{12}{ }^{13}$ The effect of these long chain fatty acids is related directly to the length of the carbon chain and the degree of unsaturation. ${ }^{12}$ Long chain polyunsaturated fatty acids (LCPUFAs) are present in breast milk, ${ }^{14}$ but until recently were not available in artificial formulas. ${ }^{15}$

Recent data from fat balance studies undertaken on infants randomised to either a formula supplemented with LCPUFAs or to an unsupplemented, but otherwise similar formula, showed that total fat absorption and the absorption of saturated fatty acids were increased in the LCPUFA supplemented group. ${ }^{16}$ It was also noted during the three day stool collection that the stools of the supplemented infants were softer than those of the unsupplemented infants. Therefore, the exclusion of LCPUFAs from formula milks may negatively influence fat hydrolysis, increase calcium soap formation, and increase stool hardness in formula fed infants.

This hypothesis has been investigated during a clinical tolerance study of an LCPUFA supplemented term formula (before its use in the investigation of the association between LCPUFA supplementation and cognitive development), and a primary objective of the double blind, randomised tolerance study was to investigate the relation of LCPUFA supplementation to stool frequency and consistency during the first 4 months of life.

Subjects and methods

Pregnant women were informed of the study after they had indicated their intention to midwifery staff that they wished to formula feed. We only recruited infants with a birth weight between 2500 and $4000 \mathrm{~g}$ and gestation between 37 and 42 weeks into our study. Infants were randomised to receive a formula that contained LCPUFAs or a formula that was unsupplemented but otherwise similar. Table 1 gives the fatty acid composition of the "ready to feed" formulas. The LCPUFA source for the supplement was egg yolk, with $\sim 70 \%$ of LCPUFAs being esters of phospholipids. The infants began feeding on the formulas immediately after delivery and the mothers received a regular supply for 4 months. Throughout our study period, mothers and researchers were 
Table 1 Fatty acid composition of the formulas with and without long chain polyunsaturated fatty acids (LCPUFAs) (g/100 g fat)

\begin{tabular}{lll}
\hline Fatty acid & With LCPUFAs & Without LCPUFAs \\
\hline C12:0 & $4.9-5.6$ & 4.8 \\
C14:0 & $5.6-5.9$ & 5.3 \\
C16:0 & $26.1-26.8$ & 25.0 \\
C18:1 n-9 & $30.2-32.2$ & 36.0 \\
C18:2 n-6 & $11.5-12.8$ & 11.4 \\
C18:3 n-3 & $0.6-0.65$ & 0.70 \\
C20:4 n-6 & $0.30-0.40$ & $<0.10$ \\
C22:6 n-3 & $0.15-0.25$ & 0.0 \\
\hline
\end{tabular}

Table 2 Sex and growth parameters at birth of the infants in the two study groups

\begin{tabular}{lll}
\hline & $\begin{array}{l}\text { LCPUFA group } \\
(n=40)\end{array}$ & $\begin{array}{l}\text { Non-LCPUFA group } \\
(n=47)\end{array}$ \\
\hline Sex $(\mathrm{M} / \mathrm{F})$ & $18 / 20$ & $23 / 24$ \\
Weight $(\mathrm{kg})$ & $3.29(0.10)$ & $3.35(0.1)$ \\
Length $(\mathrm{cm})$ & $49.9(0.4)$ & $50.3(0.3)$ \\
OFC $(\mathrm{cm})$ & $34.6(0.2)$ & $34.6(0.2)$ \\
SSSF $(\mathrm{cm})$ & $4.50(0.20)$ & $4.47(0.1)$ \\
UAC $(\mathrm{cm})$ & $10.6(0.2)$ & $10.8(0.2)$ \\
\hline
\end{tabular}

Values are mean (SEM) unless otherwise stated.

LCPUFA, long chain polyunsaturated fatty acid; OFC, occipital-frontal circumference; SSSF, subscapular skinfold thickness; UAC, upper arm circumference.

blind to the type of formula the infants received.

At the age of $1,2,3$, and 4 months a three day record of milk intake and stool pattern was recorded. The volume of formula milk was calculated by measuring all residual volumes of milk from the feeds consumed during these periods. The stool frequency and consistency were recorded during the three day periods by the mothers using standard data sheets and colour photographs of five categories of stool-watery, seedy, soft, formed, and hard. ${ }^{6} 716$

At each three day study period, the growth of the infants was assessed by the measurement of head circumference, length, weight, subscapular skinfold thickness, and mid-arm circumference.

Differences in outcomes between the groups were analysed using the Mann-Whitney U test. The study was approved by the Tayside committee on medical research ethics.

\section{Results}

Eighty eight infants were recruited to the study, 41 randomised to the LCPUFA supplemented group and 47 to the unsupplemented group. Sixty nine infants completed all four stool

Table 3 Number of hard and soft stools observed during three day stool observation periods at ages $1-4$ months

\begin{tabular}{|c|c|c|c|c|c|c|c|c|}
\hline & \multicolumn{8}{|c|}{ Age (months) } \\
\hline & \multicolumn{2}{|l|}{1} & \multicolumn{2}{|l|}{2} & \multicolumn{2}{|l|}{3} & \multicolumn{2}{|l|}{4} \\
\hline & Hard & Soft & Hard & Soft & Hard & Soft & Hard & Soft \\
\hline LCPUFA group & $\begin{array}{l}0.5 \\
(0.03)\end{array}$ & $\begin{array}{l}5.8 \\
(0.01)\end{array}$ & $\begin{array}{l}0.15 \\
(0.02)\end{array}$ & $\begin{array}{l}4.6 \\
(0.08)\end{array}$ & $\begin{array}{l}0.54 \\
(0.03)\end{array}$ & $\begin{array}{l}5.0 \\
(0.08)\end{array}$ & $\begin{array}{l}1.6 \\
(0.06)\end{array}$ & $\begin{array}{l}3.5 \\
(0.08)\end{array}$ \\
\hline Non-LCPUFA group & $\begin{array}{l}1.7 \\
(0.09)\end{array}$ & $\begin{array}{l}6.1 \\
(0.1)\end{array}$ & $\begin{array}{l}1.1 \\
(0.05)\end{array}$ & $\begin{array}{l}5.2 \\
(0.08)\end{array}$ & $\begin{array}{l}1.3 \\
(0.05)\end{array}$ & $\begin{array}{l}4.5 \\
(0.06)\end{array}$ & $\begin{array}{l}1.5 \\
(0.06)\end{array}$ & $\begin{array}{l}4.4 \\
(0.08)\end{array}$ \\
\hline p Value & 0.003 & NS & 0.001 & NS & 0.004 & NS & NS & NS \\
\hline
\end{tabular}

Values are mean (SEM)

LCPUFA, long chain polyunsaturates acid; NS, not significant. studies and 83 completed three stool studies. There were no significant differences in sex, gestation, social class, or growth parameters at birth between the two feeding groups (table 2). The milk intakes and the growth parameters at $1,2,3$, and 4 months did not differ between the two study groups (data not presented). Examination of 1905 stools (858 from LCPUFA supplemented infants, 1047 from nonsupplemented infants) was undertaken. The mean (SEM) number of stools passed for each three day study period was significantly less in the LCPUFA supplemented group (5.5 (0.3) $v$ $6.2(0.2) ; p<0.05)$. In both groups, there was a significant reduction in the number of stools passed with age $(p<0.001)$. For the analysis of the consistency of the stools, the data were grouped into two categories: (1) hard, summation of stools classified from the charts as hard or formed; and (2) soft, summation of stools classifies as soft, seedy, and watery. During the first 3 months there were significantly fewer hard stools in the LCPUFA formula group compared with the unsupplemented group (table 3). During this period, the mean (SEM) percentage of hard stools passed by infants in the LCPUFA supplemented group was 7.7 (2.1) compared with 19.2 (2.8) for unsupplemented infants $(\mathrm{p}=0.001)$.

\section{Discussion}

In our study of term infants, the pattern of decreasing stool frequency during the first 4 months of life was similar to previously reported data ${ }^{6}$; however, the randomisation of LCPUFA supplemented and nonsupplemented formulas showed that infants who received LCPUFA passed fewer stools each day and had fewer hard stools compared with infants who received the unsupplemented but otherwise identical formula. These differences existed during the first 3 months of life when most infants had not yet started eating solid foods.

The study groups consisted of full term healthy infants with no notable medical problems. The randomised design of the study ensured that there were no clinical, demographic, social, or anthropometric differences between the two study groups at birth. In addition, milk intakes were measured at monthly intervals during the first 4 months of life and the intakes did not differ at any time between groups. Data on solid feeding were not specifically recorded and it is assumed that with the randomised design of the study, the introduction of solid foods was similar for the two groups. Throughout the study, parents and investigators were blind to the formulas allocated to each infant, thus reducing the possibility of bias.

The use of photographs to classify stool hardness has been used successfully in previous studies, ${ }^{6716}$ and it has been reported that visual stool hardness correlates well with calcium soap formation. ${ }^{3}$ Calcium soaps are formed when residual saturated fatty acids precipitate with calcium in the intestinal lumen, ${ }^{3-5}$ and the increased prevalence of hard stools in formula 
fed infants is thought to reflect less efficient absorption of fat in formula fed infants compared with infants receiving breast milk. ${ }^{3}$ Breast fed infants have more efficient fat absorption because of the availability of bile salt stimulated lipase in maternal milk, ${ }^{8}$ and also because the chemical structure of triacylglycerol in breast milk is thought to be more favourable to fat digestion and absorption. ${ }^{9-11}$ In our study of formula fed infants, neither group was a recipient of bile salt stimulated lipase, and the triacylglycerol configuration did not differ significantly between the supplemented and non-supplemented formulas. There was a minor variation in C14:0 and C16:0 between the two study formulas, but if the marginal increase in saturated fats in the supplemented formula was important, the expected effect would have been an increase in prevalence of hard stools in the supplemented infants.

The mechanism underlying the passage of fewer hard stools in LCPUFA supplemented infants remains speculative. A simple explanation may be that the LCPUFA supplement, which is predominantly in the form of phospholipids, is exerting its effect through its emulsifying properties. ${ }^{17}$ The effects may also be related to the fatty acid composition of the enterocyte membrane being altered by LCPUFA supplementation, and animal studies have shown that polyunsaturated fatty acid enriched diets are associated with increased water, electrolyte, and lipid absorption. ${ }^{18}$

Alternatively, LCPUFAs may have a more specific role in intestinal fat hydrolysis. Animal studies have shown that long chain fatty acids released from triacylglycerols by the action of preduodenal lipase, ${ }^{12}$ or from phospholipids by pancreatic phospholipase $A_{2}$, might influence the binding of pancreatic colipase dependent lipase to the fat globule membrane. The enforced binding that is achieved with long chain fatty acid acting as a cofactor enables the enzyme to penetrate the fat globule membrane and hydrolyse the core triacylglycerol of the fat globule. Experimental evidence indicates that the binding activity of colipase dependent lipase is related directly to the length of the carbon chain and the degree of unsaturation of available fatty acids. ${ }^{12}$ Breast milk contains LCPUFAs ${ }^{14}$; however, until recently formula milks have been devoid of the longer chain polyunsaturated fatty acids. ${ }^{15}$ Therefore, the relative deficiency of LCPUFAs in formula milks might be associated with a reduction of pancreatic colipase dependent lipase binding activity and fat hydrolysis. In our study, the LCPUFA source for the formula supplement was egg yolk, with $\sim 70 \%$ of LCPUFA being esters of phospholipids, whereas in breast milk the LCPUFAs are predominantly in the triacylglycerol fraction. This variation in source of LCPUFAs is unlikely to alter the interpretation of our results because the hydrolysis and absorption of LCPUFAs bound to phospholipids is similar to that of triacylglycerol derived LCPUFAs. ${ }^{19}$

Recent human clinical studies have provided evidence indicating that LCPUFA supplementation influences fat absorption and stool consistency. ${ }^{14}$ Fat balance studies on 10 day old term infants who received similar trial formulas to those used in our study, demonstrated increased fat absorption in the LCPUFA supplemented group, and in particular there was increased absorption of the saturated fatty acids C14:0, C16:0, and C18:0 ${ }^{14}$ In that study, it was also noted that during the three day balance studies the supplemented infants had softer stools. The stool charts were identical to those used in our study and the frequency of hard stools in their study groups was comparable with our data (4\% in supplemented and $25.7 \%$ in unsupplemented infants).

Constipation is a common problem in childhood, accounting for $3 \%$ of paediatric outpatient visits and $16 \%$ of referrals to a specialist gastroenterology clinic. ${ }^{21}$ Although an organic cause is rarely found, the condition can be long lasting. ${ }^{21}$ It has been reported that a history of painful defecation frequently precedes chronic faecal impaction, and that early treatment of painful defecation in infancy might reduce the incidence of chronic constipation in school age children. ${ }^{2}$ During infancy, the painful passage of hard stools is a problem that is almost exclusive to formula fed infants, and evidence from this study indicates that the prevalence of hard stools is significantly reduced in infants fed a formula that is supplemented with LCPUFAs.

We acknowledge the support from Milupa Limited, UK.

1 Potts MJ, Sesney J. Infant constipation: maternal knowledge and beliefs. Clin Pediatr 1992;31:143-8.

2 Partin RM, Hamill SK, Fischel JE, Partin JS. Painful defaecation and fecal soiling in children. Pediatrics 1992;89: 1007-9.

3 Quinlan PT, Lockton S, Irwin J, Lucas AL. The relationship between stool hardness and stool composition in breastand fromula-fed infants. I Pediatr Gastroenterol Nutr and fromula-fed

4 Jandacek RJ. The solubilization of calcium soaps by fatty acids. Lipids 1991;26:250-3.

5 Mattson FH, Nolen GA, Webb MR. The absorbability by Mattson $\mathrm{FH}$, Nolen GA, Webb MR. The absorbability by
rats of various triglycerides of stearic and oleic acid and the effect of dietary calcium and magnesium. F Nutr 1979;109: $1682-7$

6 Weaver LT, Ewing G, Taylor LC. The bowel habit of milkfed infants. F Pediatr Gastroenterol Nutr 1988;7:568-71.

7 Weaver LT, Lucas AL. Development of bowel habit in preterm infants. Arch Dis Child 1993;68:317-20.

8 Hernell O. Human milk lipases. III. Physiological implications of the bile salt-stimulated lipase. Eur f Clin Invest 1975;5:267-74

9 Lien EL. The role of fatty acid composition and positional distribution in fat absorption in infants. $\mathcal{F}$ Pediatr 1994;125: S62-8.

10 Carnielli VP, Luijendijk IHT, van Goudoever JB, et al. Feeding premature newborn infants palmitic acid in amounts and stereoisomeric position similar to that of human milk: effects on fat and mineral balance. Am $\mathcal{f}$ Clin Nutr 1995;61:1037-42.

11 Lucas A, Quinlan P, Abrams S, Ryan S, Meah S, Lucas PJ. Randomised controlled trial of a synthetic triglyceride milk formula for preterm infants. Arch Dis Child 1997;77:F17884.

12 Bernback S, Blackberg L, Hernell O. Fatty acids generated by gastric lipase promote human milk triacylglycerol digestion by pancreatic colipase-dependent lipase. Biochim Biophys Acta 1989;1001:286-93.

13 Borgstrom B. Importance of phospholipids, pancreatic phospholipase $\mathrm{A}_{2}$ and fatty acid for the digestion of dietary fat. In vitro experiments with the porcine enzymes. Gastroenterology 1980;78:954-62.

14 Jensen RG. Lipids in human milk-composition and fat soluble vitamins. In: Lebenthal E, ed. Textbook of gastroenterology in infancy. New York: Raven, 1989:157-208.

15 Koletzko B, Bremer HJ. Fat content and fatty acid composition of infant formulas. Acta Paediatr Scand 1989;78:51321 . 
16 Morgan C, Davies L, Corcoran F, et al. Fatty acid balance studies in term infants fed formula milk containing long-chain

17 Watkins JB. Lipid digestion and absorption. Pediatrics 1985 ; 75(suppl): $151-6$

18 Thomson ABR, Keelan M, Clandinin MT. Onset and persistence of changes in intestinal transport following dietary fat manipulation. Lipids 1987;22:22-7.
19 Carnielli VP, Verlato G, Pederzini F, et al. Intestinal absorption of long-chain polyunsaturated fatty acids in preterm infants fed breast milk or formula. Am f Clin Nutr 1998;67: 97-103.

0 Loening-Baucke V. Chronic constipation in children. Gastroenterology 1993;105:1557-64.

21 Loening-Baucke V. Constipation in early childhood: patient characteristics, treatment and longterm follow up. Gut 1993;34:1400-4. 